\title{
A Point Prevalence Survey of Antimicrobial Prescribing in a South Indian Tertiary Hospital; Using Global PPS Tool
}

\author{
Vineela Chadalavada', Manohar Babu S. ${ }^{2}$ and Balamurugan K. ${ }^{*}$ \\ 'Department of Pharmacy, Annamalai University, Annamalai Nagar, Chidambaram - 608002, \\ Tamil Nadu, India; vineelach99@gmail.com, placementbala@yahoo.co.in \\ 2Deparment of Pharmacy, Sims College of Pharmacy, Mangaldas Nagar, Guntur - 522001, India; \\ manoharsitty@yahoo.co.in
}

\begin{abstract}
Identifying the targets for improving antimicrobial prescription and development of antimicrobial stewardship interventions can better serve to combat the situation of antimicrobial resistance. The primary objective was to determine the prevalence of antimicrobial use at tertiary care teaching hospital with both acute and long-term care patients. A periodical point prevalence survey was done in a tertiary care hospital of South India during 2019 using manual and webbased GLOBAL-PPS tool. Of 945 patients eligible for the study, 645 (69.5\%) received at least one antibiotic, with highest rates in the adult surgical ward and pediatrics. Of 645 therapeutic antibiotic prescriptions, 58.6\% prescriptions have an infectious indication of them, $62.6 \%$ are community-acquired infections. Third-generation cephalosporins were the most prescribed antibiotics. The prevalence of antibiotic use is very high, and our study evidence that the country needs a robust antimicrobial stewardship intervention program.
\end{abstract}

Keywords: Antimicrobial Prescribing, Antimicrobial Resistance, Antimicrobial Stewardship, GLOBAL-PPS, India

\section{Introduction}

Antimicrobial drugs have revolutionized the treatment of infectious diseases, becoming the cornerstone of therapy for infectious diseases to reduce morbidity and mortality ${ }^{1}$. However, there is increasing Antimicrobial Resistance (AMR) as a result of their overuse, which has become a serious problem worldwide ${ }^{2}$. India is the world's largest consumer of antibiotics for human health, which was reported 12.9 x 109 units (10.7 units per person) in $2010^{3}$. The Indian Government has issued a National Policy for Containment of Antimicrobial Resistance (AMR) to promote surveillance on antimicrobial use in the community and hospitals settings across the country. Data about the quantity and quality of antimicrobial prescribing constitute the cornerstone for guiding antimicrobial stewardship (ASP's) interventions ${ }^{4}$. India has implemented certain measures to control irrational antimicrobial use; "red strip labelling of antimicrobial packages" which indicates the drug should not be dispensed without a legal prescription ${ }^{5}$.

Antimicrobial consumption data of 65 countries presented in the WHO report for 2015-18, found wide discrepancies in consumption rates between countries. However, no countries from south-east Asia, including India, have submitted their report while efforts are initiated and ongoing in such countries with the national surveillance programs. Initial reports from India stated a very high level of consumption of third-generation cephalosporins in all its states. The WHO reported India as one of the countries for high irrational antimicrobial

${ }^{*}$ Author for correspondence 
use and inadequate surveillance and high rates of drug resistance ${ }^{6}$.

Point Prevalence Survey (PPS) in a structured qualitative assessment of Antimicrobial consumption at a given point of time. It is a feasible method to access data on antimicrobial use and helps to target improvement in the quality of antimicrobial prescribing and establish interventions for better ASH. Periodical PPS serve better $\mathrm{ASH}$ and assist in the fight against $\mathrm{AMR}^{7}$. PPS is a based stewardship tool in a few developed countries, but in other countries across the globe, clinicians have just begun to understand and explore how to use $\mathrm{it}^{7-8}$. In India, very few Point prevalent surveys of antibiotic use were reported, and most of them are registered in pediatrics ${ }^{7-12}$. To our knowledge; our present study is probably the first from Andhra Pradesh state of South India. The data gives an insight into the pattern of antimicrobial use and help in improvements.

\section{Materials and Methods}

\subsection{Study Settings and Design}

Global PPS method was adopted for the present point prevalence survey on antibiotic use at a tertiary care hospital. Point prevalence study (PPS) carried out periodically during January, May, August and December 2019 in a tertiary care hospital from Andhra Pradesh state of South India. It is a private multispecialty hospital (300 beds) located in the of Vijayawada, Andhra Pradesh province in South India. The present PPS study was designed and conducted using a structured web-based Global-PPS tool. The study tools were prepared based on point prevalence survey methodology on antibiotic use in hospitals from WHO- version 1.1 as a reference tool ${ }^{13,14}$.

The Institutional ethical committee at Andhra Hospitals initially approved the study proposal. The study is a non-experimental surveillance study with minimal risk; however, written informed consent was obtained from the patient or legal guardian under International Conference on Harmonization-Good Clinical Practice (ICH GCP) guidelines.

\subsection{Inclusion and Exclusion Criteria}

In the hospital, all the in-patients admitted at 8 am receiving at least one antimicrobial, for at least one clinical condition, prophylaxis on the day of the survey was included for the study. A patient receiving an antibiotic, e.g., every 48 hours but not receiving this antibiotic on the survey day was considered ongoing antimicrobial treatment. New-born healthy children on a maternity ward receiving antibiotics were encoded as a Neonatal Medical Ward (NMW) and included in the study. Surgical ward was surveyed on the day following the day when most elective surgical interventions usually take place or have been planned. Data was not collected from the out patients, patients discharged before 8 oclock and admitted with intervention after that study time are excluded from the study.

\subsection{Sample Size}

The estimated sample size necessary for the study was obtained using sample size calculator as 377 , where ' $p$ ' was assumed to be $50 \%$, allowable error ' $e$ ' was $5 \%$ and ' $z$ ' being the standard normal deviate with a value of $1.96^{15}$. To conduct any drug utilization studies, a sample size of at least 100 subjects was suggested by the world health organization $(\mathrm{WHO})^{16}$. However in our study, a sample size of 645 was evaluated to obtain the outcomes.

\subsection{Data Collection}

Data of antibiotic use patterns were collected from the hospital on a single day following the ESAC and GlobalPPS audit tool guidelines, also known as PPS tool ${ }^{17}$.

A well-structured data collection forms and definitions of various variables are available at GlobalPPS website; ward data collection form and patient data collection form were primarily used for the survey Antimicrobials were classified using the World Health Organization's (WHO) Anatomic Therapeutic Chemical (ATC) classification ${ }^{16}$. The ATC groups included as antimicrobial are J01 (Antibacterial for systemic use), J02 (Antimitotics), J04A (Anti-tubercular drugs), A07AA (Antibiotics for intestinal infections), D01BA (Antifungals), J05 (Antiviral's), P01AB (Antiprotozoals) and P01B (antimalarials).

Additional quality indicators included; the documented indication before the initiation of treatment; the compliance of prescription to the standard guidelines on antibiotic selection; if a stop or review information entered in the notes; prophylaxis, empiric or targeted treatment (based on microbiological data); the use of a biomarker of infection (e.g., C reactive protein) to inform antibiotic treatment was noted. Further information 
on the definitions used in the Global-PPS protocol is available online ${ }^{17}$.

In the first three months, the data from data collection forms were verified before entering into a validation form. Analysis of data was performed using SPSS version 16.0 software. In the month of December, our hospital got registered in Global-PPS web-based application system, where the present participating hospital data is being freely entered. The Global -PPS website team performed statistical analysis for the month of December and generated a report on complete antimicrobial use at our hospital during the respective period. The data from this report was pooled and utilized for further analysis.

\section{Results}

Out of 945 patients admitted with various indications in the hospital during survey days, 645 (69.5\%) received at least one antibiotic on the day of PPS. The highest antimicrobial use was evident in the adult surgical ward (78.8\%) pediatric $(61.1 \%)$ and neonatal medical wards (64\%) (Table 1).

Table 1. Overall antimicrobial prevalence

\begin{tabular}{|c|c|c|}
\hline & Total (N) & $\begin{array}{c}\text { Treated patients } \\
\text { (\%) }\end{array}$ \\
\hline $\begin{array}{c}\text { No. of hospitalised } \\
\text { patients }\end{array}$ & 945 & $645(69.5)$ \\
\hline \multicolumn{2}{|c|}{ Antimicrobial prevalence in the medical wards } \\
\hline $\begin{array}{c}\text { AMW = Adult } \\
\text { Medical Wards }\end{array}$ & 455 & $330(72.5)$ \\
\hline $\begin{array}{c}\text { P-AMW } \\
\text { Pneumology AMW }\end{array}$ & 40 & $40(100)$ \\
\hline $\begin{array}{c}\text { ASW = Adult Surgical } \\
\text { Ward }\end{array}$ & 180 & $40(78.8)$ \\
\hline $\begin{array}{c}\text { AICU = Adult } \\
\text { Intensive Care Unit }\end{array}$ & 130 & $55(61.1)$ \\
\hline $\begin{array}{c}\text { PMW = Pediatric } \\
\text { Medical Ward }\end{array}$ & 90 & $50(100)$ \\
\hline $\begin{array}{c}\text { NICU = Neonatal } \\
\text { Intensive Care Unit }\end{array}$ & 50 & $40.7)$ \\
\hline
\end{tabular}

* Patients $(\mathrm{N})=$ number of admitted adults.

Treated patients $(\%)=100^{\star}$ (number of adults treated with at least one antimicrobial/number of admitted adults).

Of 645 therapeutic antibiotic prescriptions, 378 (58.6\%) prescriptions have an infectious indication of them, $237(62.6 \%)$ are community-acquired infections, and $37.3 \%$ are hospital-acquired infections while 267 (41.4\%) prescriptions were issued for medical (28.4\%), surgical $(67.4 \%)$ prophylaxis and $4.1 \%$ targeted therapy. Among the top ten diagnoses for which antibiotics were prescribed gastrointestinal infections (26.9\%) and bacteremia (23.5\%) account for the highest rate. According to the ATC classification system, antibacterial for systemic use (J01) accounted for $82.3 \%$ (Table 2).

Table 2. General characteristics and antibiotic prescription patterns of patients surveyed

\begin{tabular}{|c|c|c|}
\hline \multicolumn{3}{|c|}{ Indications for antimicrobial prescription } \\
\hline $\mathrm{N}=645$ & No. Of patients & Percentage (\%) \\
\hline $\begin{array}{l}\text { Infections }(\mathrm{n}=\mathbf{3 7 8}) \\
\mathbf{( 5 8 . 6 \% )} \\
\text { Community- } \\
\text { acquired infection }\end{array}$ & 237 & 62.6 \\
\hline $\begin{array}{l}\text { Hospital-acquired } \\
\text { infection }\end{array}$ & 141 & 37.3 \\
\hline $\begin{array}{l}\text { Prophylaxis } \\
(\mathrm{n}=267)(\mathbf{4 1 . 4 \% )} \\
\text { Surgical prophylaxis }\end{array}$ & 180 & 67.4 \\
\hline Medical Prophylaxis & 76 & 28.4 \\
\hline Targeted therapy & 11 & 4.1 \\
\hline \multicolumn{3}{|c|}{ Top Infection for which antibiotics are prescribed $n=378$} \\
\hline Bacteremia & 89 & 23.5 \\
\hline $\begin{array}{l}\text { Gastro-intestinal } \\
\text { infections }\end{array}$ & 102 & 26.9 \\
\hline $\begin{array}{l}\text { Infection of central } \\
\text { nervous system }\end{array}$ & 18 & 4.7 \\
\hline $\begin{array}{l}\text { Intra-abdominal } \\
\text { sepsis }\end{array}$ & 31 & 8.2 \\
\hline $\begin{array}{l}\text { Pyrexia of unknown } \\
\text { origin }\end{array}$ & 52 & 13.7 \\
\hline $\begin{array}{l}\text { Bone/joint } \\
\text { infections }\end{array}$ & 36 & 9.5 \\
\hline $\begin{array}{l}\text { Fever neutropaenic } \\
\text { patient }\end{array}$ & 16 & 4.2 \\
\hline Pneumonia & 21 & 5.5 \\
\hline Other & 13 & 3.4 \\
\hline \multicolumn{3}{|l|}{$\begin{array}{l}\text { Antimicrobial } \\
\text { prescription } \\
\text { patterns }\end{array}$} \\
\hline Parenteral therapy & 531 & 82.3 \\
\hline $\begin{array}{l}\text { Multiple ATB } \\
\text { diagnosis }\end{array}$ & 237 & 36.8 \\
\hline $\begin{array}{l}\text { Multiple ATB } \\
\text { patient }\end{array}$ & 364 & 56.4 \\
\hline
\end{tabular}


${ }^{*}$ Multiple ATB diagnosis is defined as receiving $>1$ antibiotic (J01) for a single identified reason to treat (=diagnose code) at patient level.

Multiple ATB patients are defined as receiving $>1$ antibiotic (J01) at patient level.

Third-generation cephalosporins (26.2\%) were the most commonly prescribed antibiotic groups, followed by penicillins (13.5\%), quinolones (9.8\%). (Table 3, Figure 1). The distribution of various classes of antibiotics in medical, surgical, ICU and pediatric units, was depicted in (Figure 2). Among the different antimicrobial agents prescribed for systemic use (J01 ATC), the overall most frequently prescribed antibiotics were ceftriaxone (28.4\%), meropenem (7.8\%), Amikacin (7\%) and pipercillin with a beta-lactamase inhibitor (6.5\%) and levofloxacin (11.7\%); these five antibiotics accounted for 396 (61.4\%) prescriptions (Table 4) (Figure 3). Antimicrobial quality indicators presented in the hospital are shown in the (Table 5). The reason for antimicrobial use recorded for $89 \%$ medical, $100 \%$ surgical and $74.4 \%$
ICU prescriptions. The stop/review date was entered for only $26 \%$ medical and $12 \%$ surgical prescriptions. While, $43 \%$ medical, $44 \%$ surgical, $26 \%$ of ICU prescriptions are found in compliance with the hospital antimicrobial guidelines (Table 5).

\section{Discussion}

The present study is probably the first study describing antimicrobial use in Andhra Pradesh state in India using a point prevalence survey (Global-PPS). A PPS served as an inexpensive, convenient antimicrobial consumption surveillance system. The number of patients increased over time, as did the proportion treated with an antimicrobial ${ }^{18}$. Such a trend is a challenge to the hospitals surveyed and indicates that growing efforts are needed from hospital staff. There is no debate that the rate of antimicrobial prescribing in India is high compared to many other countries, which is a concern that requires addressing urgently.

Table 3. Overall proportion of antimicrobial agents prescribed

\begin{tabular}{|c|c|c|c|c|c|}
\hline & Total (\%) N=645 & Medical (\%) & Surgical (\%) & ICU/ (\%) & $\begin{array}{c}\text { Paediatric } \\
\text { NICU (\%) }\end{array}$ \\
\hline Tetracyclines (J01AA) & 4 & 3.1 & $\mathbf{0}$ & 0.9 & $\mathbf{0}$ \\
\hline Penicillin's & 13.5 & 6.6 & 0 & 2.4 & 4.5 \\
\hline $\mathrm{J} 01 \mathrm{C}$ & 6.3 & 3.8 & $\mathbf{0}$ & 0.9 & 1.6 \\
\hline J01CR & 6.5 & 2.1 & 0 & 1.5 & 2.9 \\
\hline Other beta-lactams & 42.2 & & & & \\
\hline J01DB & 0 & $\mathbf{0}$ & $\mathbf{0}$ & $\mathbf{0}$ & $\mathbf{0}$ \\
\hline J01DC & 1.8 & 1.8 & $\mathbf{0}$ & 0 & $\mathbf{0}$ \\
\hline J01DD & 26.2 & 15.2 & 3.2 & 4.4 & 3.4 \\
\hline J01DF & 0.9 & 0.9 & $\mathbf{0}$ & $\mathbf{0}$ & $\mathbf{0}$ \\
\hline $\mathrm{J} 01 \mathrm{DH}$ & 8.2 & 3.2 & $\mathbf{0}$ & 3.2 & 1.8 \\
\hline J01FA & 5.1 & 2.1 & 1.3 & 1.1 & 0.6 \\
\hline J01GB & 7 & 2.4 & 0 & 1.3 & 3.3 \\
\hline J01MA & 9.8 & 5.4 & 2 & 0.3 & 2.1 \\
\hline P01CA & 5.2 & 3 & $\mathbf{0}$ & 1.8 & 0.4 \\
\hline Others & 5.5 & 2.3 & 0.2 & $\mathbf{0}$ & 1.2 \\
\hline
\end{tabular}

Tetracyclines (J01AA), Penicillins with extended spectrum (J01CA), Combinations of penicillins, incl. beta-lactamase inhibitors (J01CR), Second-generation cephalosporins (J01DC), Third-generation cephalosporins (J01DD), Fourth-generation cephalosporins (J01DF), Carbapenems (J01DH), Macrolides (J01FA), Lincosamides (J01FF), Aminoglycosides (J01GB), Quinolones (J01MA), Nitroimidazole Derivatives (P01CA) 


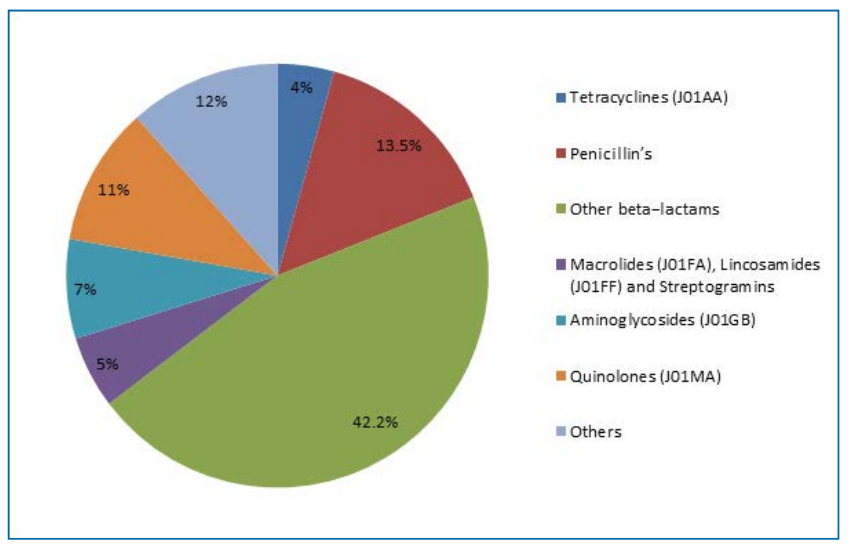

Figure 1. Portion of antibiotic use in the study settings.

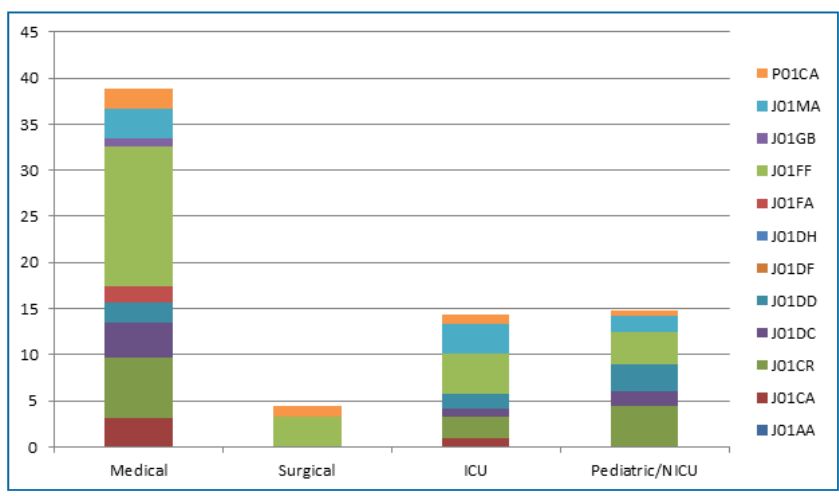

Tetracyclines (J01AA), Penicillins with extended spectrum (J01CA), Combinations of penicillins, incl. beta-lactamase inhibitors (J01CR), Second-generation cephalosporins (J01DC), Third-generation cephalosporins (J01DD), Fourth-generation cephalosporins (J01DF), Carbapenems (J01DH), Macrolides (J01FA), Lincosamides (J01FF), Aminoglycosides (J01GB), Quinolones (J01MA), Nitroi midazole Derivatives (P01CA)

Figure 2. Antimicrobials prescribed by classification.

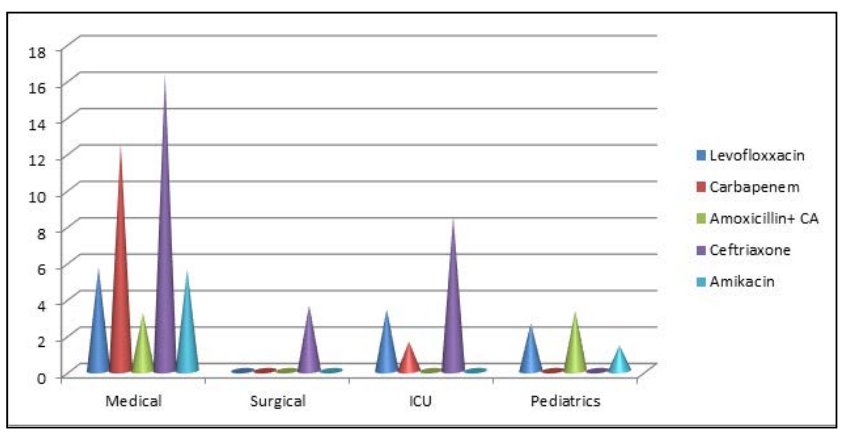

Figure 3. Top 5 most frequently used prescribed antibiotics in adults and children.
We report a higher rate of antimicrobial use (62.6\%) similar to few studies from India $51.6 \%$ \& $61.5 \%$ \& $64.74 \%^{7,9-10}$. PPS studies from various countries reported antimicrobial use; French (32.2\%), Sweden (33.3\%), turkey (30.6\%), Jordan (46.2\%), Belgian (27.1\%), Brazil (52.2\%), Nigeria (69.7\%), Switzerland (33\%) Canada (65\%), Egypt (59\%), Saudi (46.9\%) and China $(56 \%)^{19-30}$. However, this overall prevalence masks important regional differences: 2017 data showed that among 68 hospitals in East and South Asia the prevalence of antimicrobial use was $48.2 \%$, compared with $29.6 \%$ in 106 European hospitals. There have been few previous studies in India. However, our data are comparable to a study in Eastern India, which reported antimicrobial use prevalence of $62 \%$ in 2014 and $69.1 \%$ in $2017^{8}$. From (figure 2), the highest prevalence of antibiotic use in the present study was in surgical and pediatrics while it was in Gynecology ward reported in a similar study done at south India?

In our study, a significant portion of antibiotics was mostly used for the treatment of community-acquired infections (62.6\%), and these results are similar to studies in Nigeria and Sweden and to two other studies conducted in India, which reports community acquired infections (CAI) as an indication with the highest prevalence of antimicrobial use ${ }^{7,12}$. The clinical sign documented for the treatment with antimicrobials is vital to evaluate compliance with national antimicrobial treatment guide lines (ICMR Guidelines).

On reviewing empirical antimicrobial therapies with CAI, the two most common antimicrobials were third generation cephalosporins (26.2\%) (Table 3) which is similar to two other Indian studies ${ }^{7,11}$. The similar wide use of cephalosporins was reported in Turkey, Jordan, Brazil, Nigeria and Saudi. However, in European countries, wide use of Fluoroquinolones in French and Belgian, Amoxicillin/Clavulanic acid combination in Northern Ireland and Switzerland were reported ${ }^{18-29}$. Guidelines recommend the use of third-generation Cephalosporins only when first-line agents are ineffective ${ }^{31}$. Hence this study identified an opportunity to improve antimicrobial use by prescribing first-line drugs for hospitalized CAI patients.

Some poor-prescribing practices such as indications for the therapy and low documentation rate of stop or review dates to guide the scheduled antimicrobial therapy may result in healthcare staff administering medication for the inappropriate duration (Table 5). The condition might be due to lack of quality-assured procedures and 
Table 4. Top 5 most frequently used prescribed antibiotics in adults and children

\begin{tabular}{|c|c|c|c|c|c|}
\hline Antibiotic & $\begin{array}{c}\text { Medical (\%) } \\
\mathbf{N}=330\end{array}$ & $\begin{array}{c}\text { Surgical (\%) } \\
\mathbf{N}=180\end{array}$ & $\begin{array}{c}\text { ICU/ (\%) } \\
\text { N=130 }\end{array}$ & $\begin{array}{c}\text { Paedi } \\
\text { atric/ NICU (\%) } \\
\text { N=140 }\end{array}$ & Total \\
\hline Levofloxacin & 5.7 & 0 & 3.4 & 2.6 & $11.7 \%$ \\
\hline Meropenem & 12.5 & 0 & 1.6 & 0 & $7.8 \%$ \\
\hline Amoxicillin and Clavulanic acid & 3.2 & 0 & 0 & 3.3 & 6.5 \\
\hline $\begin{array}{c}\text { Ceftria } \\
\text { xone }\end{array}$ & 16.3 & 3.6 & 8.5 & 0 & $28.4 \%$ \\
\hline Amikacin & 5.6 & 0 & 0 & 1.4 & $7 \%$ \\
\hline
\end{tabular}

Table 5. Quality indicators of antibiotic prescribing

\begin{tabular}{|c|c|c|c|c|}
\hline \multirow{2}{*}{ Indicator } & & Medical (\%) & Surgical (\%) & ICU (\%) \\
\hline Indication for treatment was recorded & Yes & 89 & 100 & 74.4 \\
\hline \multirow{3}{*}{ Compliance with the hospital guidelines } & Compliant & 43 & 44 & 26 \\
\cline { 2 - 5 } & Not compliant & 20 & 26 & 19 \\
\cline { 2 - 5 } & Not-assessable & 21 & 18 & 13 \\
\cline { 2 - 5 } & No information & 16 & 12 & 42 \\
\hline Stop/review date documented & Yes & 26 & 64 & 0 \\
\hline Targeted therapy & Yes & 11 & 0 & 0 \\
\hline Biomarkers & Yes & 0 & 0 & 0 \\
\hline
\end{tabular}

guidelines ${ }^{32}$. Surprisingly, the majority of prescriptions comply with the hospital prescribing guidelines. In the present study settings, an antimicrobial policy was structured and made practiced at the hospital by a clinical pharmacist is much appreciable. Besides, a very high rate of parenteral use of antimicrobials was reported, contrary to the advocated practices in antimicrobial stewardship program. There was no utilization of biomarkers across the study hospital because they are novel and expensive. However, they are a viable adjunct to guide therapy in select patients such as those in whom sepsis is suspected.

Some limitations should be acknowledged. As this was a point prevalence survey, patients were not followed-up in time. This study only shows a snapshot of the situation and shallow sample size; therefore, they are not ideal for measuring, for example, Antimicrobial use (AMR). Good outcomes with antimicrobials (i.e., appropriate antimicrobial prescribing and reduction of resistance to antimicrobials) require the use of antimicrobial stewardship approaches and completion of PPS at regular intervals.

\section{Conclusion}

This study indicated a high rate of antibiotic prescription with increased usage of third-generation Cephalosporin's. These findings suggest critical areas for interventions and propose implementation of antimicrobial stewardship policies in the present study hospital. The current survey was in a singles institute, and we further suggest such periodical surveys in multiple institutions across the state for the development of strong antibiotic policies. Our study evidence the country needs strong antimicrobial stewardship intervention program.

\section{Conflicts of Interests}

No reported conflicts to declare.

\section{Funding}

No financial support was received by any of the authors for conducting this survey. 


\section{References}

1. Alharbi SA, Wainwright M, Alahmadi TA, Salleeh HB, Faden AA, Chinnathambi A. What if Fleming had not discovered penicillin? Saudi J. Biol. Sci. 1 Sep 2014; 21(4): 289-93. https://doi.org/10.1016/j.sjbs.2013.12.007

2. Laxminarayan R, Matsoso P, Pant S, Brower C, Røttingen JA, Klugman K, Davies S. Access to effective antimicrobials: a worldwide challenge. The Lancet. 9 Jan 2016; 387(10014): 168-75. https://doi.org/10.1016/S0140-6736(15)00474-2

3. Van Boeckel TP, Gandra S, Ashok A, Caudron Q, Grenfell BT, Levin SA, Laxminarayan R. Global antibiotic consumption 2000 to 2010: an analysis of national pharmaceutical sales data. The Lancet infectious diseases. 1 Aug 2014; 14(8):742-50. https://doi.org/10.1016/S14733099(14)70780-7

4. National centre for disease control. National programme on AMR containment. Assed on 20-08-2020. https://ncdc.gov. in/index1.php?lang=1\&level=2\&sublinkid=384\&lid=344.

5. Travasso C. India draws a red line under antibiotic misuse. BMJ 2016; 352: i1202. https://doi.org/10.1136/bmj. i1202

6. World Health Organization. WHO report on surveillance of antibiotic consumption: 2016-2018 early implementation. Assed on 2-11-2020. https://www.who.int/medicines/ areas/rational_use/oms-amr-amc-report-2016-2018/en/ (2018).

7. Vadivoo NS, Usha B, Sudha K. Study to assess quality of antimicrobial use by point prevalence survey at a tertiary care centre. Indian J Microbiol Res. 2019; 6(3):245-252. https://doi.org/10.18231/j.ijmr.2019.054

8. Ravi N, Laha A, Hmar L, Chatterjee S, Goswami J, Goel G, Dhar K, Ghosh T, Chatterjee S, Datta SS, Bhattacharya S. Exploring the prescribing behaviours and the mind of antibiotic prescribers is critical for a successful antibiotic stewardship programme: Results of a survey from Eastern India. Indian J. Med. Microbiol. 1 Apr 2017; 35(2):299. https://doi.org/10.4103/ijmm.IJMM_17_133

9. Gandra S, Alvarez-Uria G, Murki S, Singh SK, Kanithi R, Jinka DR, Chikkappa AK, Subramanian S, Sharma A, Dharmapalan D, Kandraju H. Point prevalence surveys of antimicrobial use among eight neonatal intensive care units in India: 2016. Int J Infect Dis. 2018 Jun 1; 71:20-4. https:// doi.org/10.1016/j.ijid.2018.03.017

10. Sharma M, Damlin A, Pathak A, Lundborg CS. Antibiotic prescribing among pediatric inpatients with potential infections in two private sector hospitals in Central India. PLoS One. 5 Nov 2015; 10(11):e0142317. https://doi. org/10.1371/journal.pone.0142317

11. Singh SK, Sengupta S, Antony R, Bhattacharya S, Mukhopadhyay C, Ramasubramanian V, Sharma A, Sahu S, Nirkhiwale S, Gupta S, Rohit A. Variations in antibiotic use across India: multi-centre study through Global Point Prevalence survey. J. Hosp. Infect. 1 Nov 2019; 103(3): 2803. https://doi.org/10.1016/j.jhin.2019.05.014

12. Singh S, Jose T, Versporten A, Sengupta S, Fini P, Sharland M, Kumar RK, Goossens H. A point prevalence surveillance study from pediatric and neonatal specialty hospitals in India. Pediatr Infect Dis J. 1 Jan 2014; 9(3):151-5. https:// doi.org/10.3233/JPI-140429

13. World Health Organization. In: and others, editor. WHO methodology for point prevalence survey on antibiotic use in hospitals, version 1.1. World Health Organization. Assessed on 2-11-2020. http://www.who.int/iris/ handle/10665/280063.

14. World Health Organization. How to investigate antimicrobial use in hospitals: selected indicators. 2012. Assed on 1-11-2020. http://apps.who.int/medicinedocs/documents/ s21031en/s21031en.pdf.

15. Raosoft. Sample size calculator. Assed on 1-11-2020. http:// www.raosoft.com/samplesize.html.

16. World Health Organization (1993) How to investigate drug use in health facilities: Selected drug use indicators. Geneva, World Health Organization 1993. WHO/DAP 1: 1-87. http://www.global-pps.com/documents

17. WHO Collaborating Centre for Drug Statistics Methodology. ATC/DDD Index 2017. Assessed on 1-112020. https://www.whocc.no/atc_ddd_index/.

18. Versporten A, Zarb P, Caniaux I, Gros MF, Drapier N, Miller M, Jarlier V, Nathwani D, Goossens H, Koraqi A, Hoxha I. Antimicrobial consumption and resistance in adult hospital inpatients in 53 countries: results of an internet-based global point prevalence survey. The Lancet Global Health. 1 Jun 2018; 6(6):e619-29.

19. Robert J, Péan Y, Varon E, Bru JP, Bedos JP, Bertrand X, Lepape A, Stahl JP, Gauzit R. Point prevalence survey of antibiotic use in French hospitals in 2009. J. Antimicrob. Chemother. 1 Apr 2012; 67(4):1020-6. https://doi. org/10.1093/jac/dkr571

20. Skoog G, Struwe J, Cars O, Hanberger H, Odenholt I, Prag M, Skärlund K, Ulleryd P, Erntell M. Repeated nationwide point-prevalence surveys of antimicrobial use in Swedish hospitals: data for actions 2003-2010. Eurosurveillance. 23 Jun 2016; 21(25):30264. https://doi.org/10.2807/15607917.ES.2016.21.25.30264

21. Usluer G, Ozgunes I, Leblebicioglu H. A multicenter point-prevalence study: antimicrobial prescription frequencies in hospitalized patients in Turkey. Annals of clinical Microbiology and Antimicrobials. Dec 2005; 4(1): 1-5. https://doi.org/10.1186/1476-0711-4-16

22. Elhajji FD, Al-Taani GM, Anani L, Al-Masri S, Abdalaziz H, Su'ad H, Al Bawab AQ, Scott M, Farren D, Gilmore F, Versporten A. Comparative point prevalence survey of 
antimicrobial consumption between a hospital in Northern Ireland and a hospital in Jordan. BMC health services research. 1 Dec 2018; 18(1):849. https://doi.org/10.1186/ s12913-018-3656-y

23. Porto AP, Goossens H, Versporten A, Costa SF, Brazilian Global-PPS Working Group. Global point prevalence survey of antimicrobial consumption in Brazilian hospitals. J. Hosp. Infect. 1 Feb 2020; 104(2):165-71. https://doi. org/10.1016/j.jhin.2019.10.016

24. Oduyebo OO, Olayinka AT, Iregbu KC, Versporten A, Goossens H, NwajiobiPrincewill PI, et al. A point prevalence survey of antimicrobial prescribing in four Nigerian tertiary hospitals. Ann Trop Pathol. 2017; 8(1):42-6. https://doi.org/10.4103/atp.atp_38_17

25. Zingg W, Metsini A, Gardiol C, Balmelli C, Behnke M, Troillet N, Widmer A, Pittet D. Antimicrobial use in acute care hospitals: national point prevalence survey on healthcare-associated infections and antimicrobial use, Switzerland, 2017. Eurosurveillance. 15 Aug 2019; 24(33):1900015. https://doi.org/10.2807/1560-7917. ES.2019.24.33.1900015

26. Lee C, Walker SA, Daneman N, Elligsen M, Palmay L, Coburn B, Simor A. Point prevalence survey of antimicrobial utilization in a Canadian tertiary-care teaching hospital. Journal of epidemiology and global health. 1 Jun 2015; 5(2): 143-50. https://doi.org/10.1016/j.jegh.2014.06.003

27. Talaat M, Saied T, Kandeel A, El-Ata GA, El-Kholy A, Hafez S, Osman A, Razik MA, Ismail G, El-Masry S, Galal R. A point prevalence survey of antibiotic use in 18 hospitals in Egypt. Antibiotics. Sep 2014; 3(3):450-60. https://doi. org/10.3390/antibiotics3030450

28. Al Matar M, Enani M, Binsaleh G, Roushdy H, Alokaili D, Al Bannai A, Khidir Y, Al-Abdely H. Point prevalence survey of antibiotic use in 26 Saudi hospitals in 2016. J. Infect. Public Health. 1 Jan 2019; 12(1):77-82. https://doi. org/10.1016/j.jiph.2018.09.003

29. Xie DS, Xiang LL, Li R, Hu Q, Luo QQ, Xiong W. A multicenter point-prevalence survey of antibiotic use in 13 Chinese hospitals. J. Infect. Public Health. 1 Jan 2015; 8(1): 55-61. https://doi.org/10.1016/j.jiph.2014.07.001

30. National treatment guidelines for antimicrobial use in infectious disease. 2016. Assessed on 01-11-2020. https:// ncdc.gov.in/WriteReadData/1892s/File622.pdf

31. Bebell LM, Muiru AN. Antibiotic use and emerging resistance: how can resource-limited countries turn the tide? Global heart. 1 Sep 2014; 9(3):347-58. https://doi. org/10.1016/j.gheart.2014.08.009

32. Absolutereports.com. Global (North America, Europe and Asia-Pacific, South America, Middle East and Africa) Procalcitonin (CAS 56645-65-9) Market 2017 Forecast to 2022. Pune; 2017. Available from: https://www.absolutereports.com/11101460 [Last accessed on 2017 Sep 25]. 